\title{
Injection-Moulding Compounding of PP Polymer Nanocomposites
}

\author{
Markus Gottfried Battisti* - Walter Friesenbichler \\ University of Leoben, Chair of Injection Moulding of Polymers, Department Polymer Engineering and Science, Austria
}

\begin{abstract}
The objective of this study is a comparison of different compounding techniques and their influence on Young's modulus. Both conventional processing of polymer nanocomposites and processing in the unique Injection Moulding Compounder (PNC-IMC) were evaluated. Additionally, the effects of nanofillers on the thermal conductivity of polymer nanocomposites were investigated at various pressures.

In comparison to conventional compounding process, in which the compound must be pelletized and fed into the injection moulding machine for the second plasticizing process, injection-moulding compounding combines these two processing steps. The material compounding and the subsequent injection moulding are done directly with only one plasticizing process, with the use of a heated melt line and a melt accumulator. In this study, both of these techniques were used for the production of polymer nanocomposites.

This paper shows the different effects of processing techniques, screw speed, counter-pressure and different extruder length on Young's modulus and demonstrates that, for the improvements, a compromise between shear energy input and residence time is essential. The increase of thermal conductivity by using nanofillers in comparison to the virgin polypropylene is shown. The investigated increase in thermal conductivity should be extremely appealing for the industry in terms of cycle time reduction in the injection moulding process.

These first results give an excellent overview of both the possibilities and the limitations of the innovative concept of the PNC-IMC.
\end{abstract} Further studies on the detailed understanding of the exfoliation and intercalation of layered silicates in polymer melt will be done.

Keywords: injection moulding compounding, polymer nanocomposites, thermal conductivity, exfoliation, intercalation, layered silicates

\section{INTRODUCTION}

\subsection{Injection Moulding Compounding}

The Injection Moulding Compounder (IMC) concept was first presented by Krauss Maffei at the K1998 for long glass-fibre-reinforced thermoplastics. Its characteristic feature is a twin-screw extruder, which is directly integrated into an injection-moulding system.

The injection-moulding compounding process combines two processing steps: the material compounding, which normally takes place at the raw material manufacturer and the injection-moulding process which usually is done at the injection moulder. The final part is processed directly with only one plasticizing process, and the material used does not have to be granulated and plasticized again after the compounding step in the plasticizing unit of an injection-moulding machine. The molten material exits the extruder and is directly fed into a melt accumulator and further into the injection unit. After the specimen volume is reached, a conventional injection-moulding process starts. The accumulator serves as a buffer element between the continuouslyoperating extruder and the discontinuously-operating injection unit. The outcome is greater flexibility for the processor when improving both the component structure and the quality of the moulded part, as well as demonstrably lower production costs [1] and [2].
To date, all presented injection-moulding compounding systems have been designed for the processing of fibres (long glass fibres, natural fibres). The benefit of processing in a "single heat" is the lower working temperature and the shorter residence time in the whole processing facility, as well as the lower reduction of the fibre length. The material's properties are adjusted by the addition of additives, for instance, to improve bonding of the polar natural fibres to the nonpolar polyolefins, which is also necessary for layered silicates. Another advantage of the one-step process compared to the two-step process is energy savings of about $40 \%$, since the more energy-efficient, continuously operating co-rotating twin-screw extruder replaces the energy-intensive step of plasticating by means of a reciprocating screw [3].

Wobbe [4] states that enormous areas remain unexplored when considering the possibilities and advantages of "single-heat" injection moulding. Examples are:

- Long fibres are shortened less (the length is responsible for the stiffness of the fibre composite) because there is no second plasticizing process in the injection unit.

- There is less wear and tear on the machine components, because a high level of abrasive fillers can be added to the molten polymer downstream.

- It will be easier to produce finished articles from compounds that have a tendency to separate.

*Corr. Author's Address: University of Leoben, Chair of Injection Molding of Polymers, Department Polymer Engineering and Science, Austria, markus.battisti@unileoben.ac.at 
The MUL (University of Leoben) polymer nanocomposite Injection Moulding Compounder (PNC-IMC) described below (Fig. 1) is the world's first specially adapted IMC for the manufacturing and injection moulding of polymer nanocomposites. It consists of a ZSE 27 MAXX Leistritz-Compounder and an $1800 \mathrm{kN}$ fully-electric injection moulding machine by Engel. A 3-way valve and its particular arrangement allow for using the IMC as three-inone production line either for injection moulding compounding, for injection moulding or as a separately acting twin-screw compounder line. Furthermore, this PNC-IMC can also be used for the homogenization of fillers in high temperature polymers (HTP) in temperatures ranging up to $400{ }^{\circ} \mathrm{C}$.

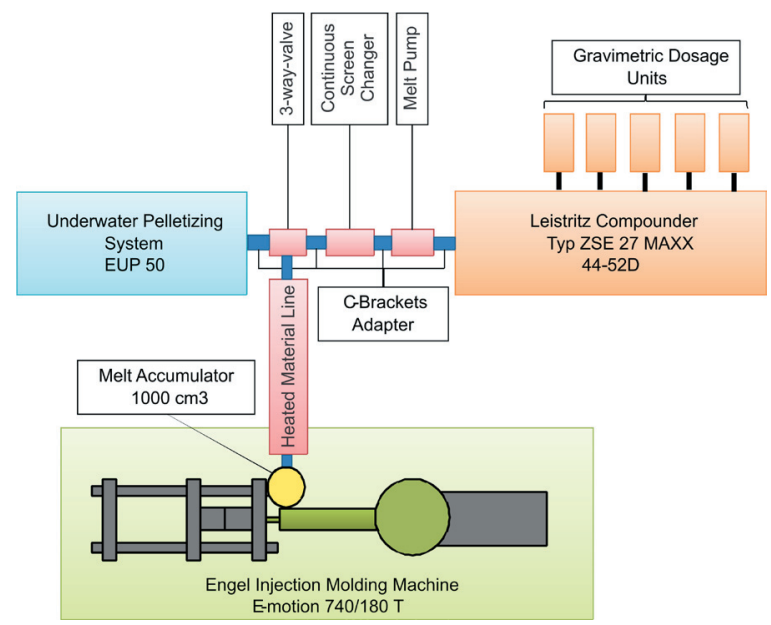

Fig. 1. System schematic of the MUL Polymer NanoComposite Injection Moulding Compounder (PNC-IMC)

\subsection{Compounding of Polymer Nanocomposites}

Compounding by melt mixing is the most attractive industrial method for the preparation of polymer nanocomposites due to its technological simplicity. Common polymer processing machines are much easier and more pleasant to use than using equipment and procedures in chemical laboratories, which are required for in situ or solution methods [5]. Furthermore, these "laboratory methods" are only useful for limited amounts of compounds; therefore, they are less desirable for many industry applications [6].

From the economical point of view, the use of a co-rotating twin-screw extruder as a continuous processing technology is preferable to melt mixing in a discontinuous kneader. High shear energy (usage of kneading blocks and high screw speed) and long residence time are necessary for the preparation of polymer nanocomposites and the successful dispersion of silicate plates in a polymer melt. Based on preliminary work at MUL, it is now possible to realize high shear energy as well as long residence time (which are normally mutually exclusive in the extrusion process) by implementing a melt pump in front of the twin-screw extruder [7]. Further possibilities for diversifying the residence time of the polymer melt are the screw length of the extruder, and the processing in an IMC, both of which are possible with the PNC-IMC described.

Our aim is the improvement of the mechanical properties [8] and materials with higher thermal conductivity, in order to reduce the cooling period of injection moulded parts and blow-moulded parts with medium-to-high wall thicknesses, respectively. There are many reasons for the use of nanoclays in polymer systems ranging from the enhancement of structural and mechanical properties to functional materials with a tailored performance profile for thermostability, flame retardance, barrier properties or enhancement of thermal conductivity [9] and [10].

\subsection{Thermal Conductivity of Polymers}

Thermal conductivity is the most commonly used property that helps to quantify the transport of heat through a material. In polymers, thermal conductivity is based on two different mechanisms: heat is transferred through Van-der-Waals forces, and phonons are stimulated through covalent bonding. By definition, energy is transported proportionally to the speed of sound; therefore, thermal conductivity follows the relationship

$$
\lambda=c_{p} \cdot \rho \cdot v \cdot l,
$$

where the thermal conductivity $\lambda$ is directly proportional to the specific heat capacity $c_{p}$, the density $\rho$, the speed of sound $v$, and the molecular separation $l$ of the elastic waves [11].

Due to the increase in density below the solidification of semi-crystalline thermoplastics, the thermal conductivity is higher in the solid state than in the melt. Furthermore, higher pressure also increases the thermal conductivity. Other factors, such as temperature, morphology and molecular orientation, are also affecting the thermal conductivity of polymers. Highly drawn semi-crystalline polymers like PP can have a much higher thermal conductivity in the direction of orientation of the polymer chains than perpendicular to it. 
It is also beneficial to know the thermal conductivity of filled compositions in order to model and analyse the heat transfer process during moulding. Thermal conductivity can be influenced significantly by the addition of different fillers and additives since the thermal conductivity of most inorganic fillers is around 10 times higher than that of polymers. The increase of thermal conductivity depends on the type, on the amount as well as on the particle shape, the size and the distribution of the used fillers [12].

\section{EXPERIMENTAL}

\subsection{Materials}

An isotactic polypropylene (PP) homopolymer Bormed DM55pharm (MFR $2.8 \mathrm{~g} / 10 \mathrm{~min} ; 230^{\circ} \mathrm{C} / 2.16$ $\mathrm{kg}$ ) used for the preparation of nanocomposites was supplied by Borealis. The used nanofiller was Nanofil 5 (montmorillonite intercalated with dimethyl distearyl ammonium chloride) and the compatibilizer Scona TPPP 2112 GA (PP grafted with $1.2 \mathrm{wt} \%$ of maleic acid anhydride, PP-g-MA, MFR 6 g/10 min; $190{ }^{\circ} \mathrm{C} / 2.16 \mathrm{~kg}$ ) was supplied by Kometra.

\subsection{Preparation of Polypropylene Nano-Composites}

The preparation of the polymer nanocomposites had been carried out with the intermeshing, co-rotating twin-screw extruder of the PNC-IMC. The possibility of varying the length of the extruder has opened a large area for scientific development and research, and practical applications. We used 11 or 13 barrel segments for the compounding process in which each barrel segment had a length of four times the screw diameter $D$. PP and compatibilizer were fed upstream through the main hopper, and the organoclay was fed downstream at the $4^{\text {th }}$ extruder barrel for both equipment configurations. All the components were fed by separately controlled gravimetric dosage units at an overall throughput rate of $6 \mathrm{~kg} / \mathrm{h}$. The formulation of the compounded polymer nanocomposites was constant at $90 \mathrm{wt} \%$ polypropylene, $5 \mathrm{wt} \%$ organoclay and $5 \mathrm{wt} \%$ compatibilizer. The screw geometry was held constant. The screw speed and the counterpressure of the melt pump were varied according to Table 1 in order to observe the influence of different processing conditions.

It is necessary to know that the pressure gradient in the melt Witte pump is negative to increase the residence time and shear rate of the polymer compound leading to improved dispersion of the nanofiller. The minimal residence time was measured as the time between the colour masterbatch granulate insertion into the hopper and the colour change of the outgoing molten string at the three-way-valve and the injected part, respectively. The extruder temperature profile was set at 170 to $210^{\circ} \mathrm{C}$ from hopper up to the EUP 50 underwater pelletizing system, which is a water process \& drying system and self-cleaning centrifuge.

Table 1. Indication of the prepared nano-composites; extruder length as multiple of the screw diameter $D$

\begin{tabular}{|c|c|c|}
\hline $\begin{array}{c}\text { Extruder length } \\
D\end{array}$ & $\begin{array}{c}\text { Screw speed } \\
\text { [rpm] }\end{array}$ & $\begin{array}{c}\text { Counter pressure } \\
\text { [bar] }\end{array}$ \\
\hline 44 & 50 & 50 \\
\hline 44 & 50 & 75 \\
\hline 44 & 50 & 100 \\
\hline 44 & 100 & 50 \\
\hline 44 & 100 & 75 \\
\hline 44 & 100 & 100 \\
\hline 44 & 150 & 50 \\
\hline 44 & 150 & 75 \\
\hline 44 & 150 & 100 \\
\hline 52 & 50 & 50 \\
\hline 52 & 50 & 75 \\
\hline 52 & 50 & 100 \\
\hline 52 & 100 & 50 \\
\hline 52 & 100 & 75 \\
\hline 52 & 100 & 100 \\
\hline 52 & 150 & 50 \\
\hline 52 & 150 & 75 \\
\hline 52 & 150 & 100 \\
\hline
\end{tabular}

In order to monitor the differences to the processing with the PNC-IMC (processing in a single heat), two compounding grades were manufactured, both conventionally with the underwater pelletizing system and in the IMC. These experiments were made both with $5 \mathrm{wt} \%$ organoclay and $5 \mathrm{wt} \%$ compatibilizer as well as $10 \mathrm{wt} \%$ organoclay and $10 \mathrm{wt} \%$ compatibilizer, to verify the influence of nanofillers on the thermal conductivity.

Another experiment was the manufacturing of polypropylene nanocomposites with a pre-stage masterbatch process. Here, a masterbatch of $50 \mathrm{wt} \%$ organoclay and $50 \mathrm{wt} \%$ compatibilizer was produced in the twin-screw extruder and then pelletized. The masterbatch pellets were fed into the twin-screw extruder again and then diluted with virgin PP to receive a compound of $5 \mathrm{wt} \%$ nanofiller and $5 \mathrm{wt} \%$ compatibilizer. 
Table 2. Various options for the production of the polypropylene nanocomposites compounds

\begin{tabular}{|c|c|}
\hline $\begin{array}{l}\text { One-step } \\
\text { process }\end{array}$ & $\begin{array}{l}\text { Production of tensile bars in a single heat } \\
\text { IMC-process (one plasticizing process) }\end{array}$ \\
\hline $\begin{array}{l}\text { Two-step } \\
\text { process }\end{array}$ & $\begin{array}{l}\text { Conventional compounding and pelletizing and } \\
\text { subsequent injection moulding of tensile bars }\end{array}$ \\
\hline $\begin{array}{l}\text { 3in1 } \\
\text { process }\end{array}$ & $\begin{array}{l}\text { - Direct compounding of the organoclay and the } \\
\text { - } \quad \text { Processibing with the one- or two-step process }\end{array}$ \\
\hline $\begin{array}{l}\text { Masterbatch } \\
\text { (MB) process }\end{array}$ & $\begin{array}{l}\text { - } \quad \text { Production of the MB (50 wt\% organoclay and } \\
50 \text { wt\% compatibilizer) } \\
\text { - } \quad \text { Dilution of the MB in the compounder with } \\
\text { PP to receive a compound with } 5 \text { or } 10 \text { wt } \% \\
\text { organoclay and compatibilizer } \\
\text { - } \quad \text { Processing with the one- or two-step process }\end{array}$ \\
\hline
\end{tabular}

\subsection{Mechanical Properties - Tensile Test}

The tensile bars for the investigation of the mechanical properties were produced both directly with the PNCIMC and in the two-step process with the injection moulding machine of the PNC-IMC. To guarantee the best and primarily the same processing conditions for all test specimens, test series were made before the test specimen's production. In the near future, the optimisation of the manufacturing process with the use of Design of Experiments (DoE) is planned [13].

Table 3. Injection moulding conditions for the production of the test specimens

\begin{tabular}{lc}
\hline $\begin{array}{l}\text { Cylinder temperature }\left[{ }^{\circ} \mathrm{C}\right] \\
(\text { Z1-Z2-Z3-Z4-ZN) }\end{array}$ & 190-200-200-210-210 \\
\hline Mould temperature $\left[{ }^{\circ} \mathrm{C}\right]$ & 40 \\
\hline Holding pressure $[\mathrm{bar}]$ & 800 \\
\hline Injection speed [cm $\left.{ }^{3} / \mathrm{s}\right]$ & 75 \\
\hline Dosing speed $[\mathrm{m} / \mathrm{s}]$ & 0.075 or IMC \\
\hline
\end{tabular}

A Zwick Z010 universal tensile testing machine was used to carry out the tensile tests according to standard ISO 527-1. All tests were done at standardized conditions $\left(23 \pm 2{ }^{\circ} \mathrm{C} / 50 \pm 5 \%\right.$ r.H. $)$. The data was evaluated using the testXpert II software.

\subsection{Thermal Conductivity}

The thermal conductivity measurements based on the Line-Source method according to ASTM D5930-97 in a temperature range from 80 to $240{ }^{\circ} \mathrm{C}$ were carried out using a high pressure capillary rheometer with a single channel system having a diameter of $15 \mathrm{~mm}$. At the beginning of each measurement, the high pressure capillary rheometer was heated to the specified temperature for 60 minutes. A defined weight of the specimen was filled in the cylinder and compressed tightly while filling. Afterwards, the material was compressed with a piston for 10 minutes and then the measurement started at the defined pressure stage. At least three measurements for each temperature were made, with a 12- to 13-minute delay between each measurement. While the specimens were cooling, the waiting time between each temperature range was 20 to 30 minutes.

\section{RESULTS AND DISCUSSION}

\subsection{Effects of Screw Speed, Counter-Pressure and Different Extruder Length on Young's Modulus}

As explained earlier in this paper, the shear energy and residence time are the two main influential variables, which normally act opposite in the extrusion process. The effects of screw speed and counter-pressure on Young's modulus for an extruder length of 44D are plotted in Fig. 2. It is obvious that Young's modulus decreases with increasing screw speed. The most significant decline occurs from 100 to $150 \mathrm{rpm}$. That means with higher screw speed and thereby higher shear forces, the quality of the polymer melt is reduced. Increasing counter-pressure of the melt pump shows a similar effect. At 50 and $100 \mathrm{rpm}$ screw speed, Young's modulus decreases almost linearly; only at $150 \mathrm{rpm}$ is a plateau at $75 \mathrm{bar}$ reached. Young's modulus values of pure PP result from tensile tests of injection-moulded parts, which were produced conventionally without an injectionmoulding compounding process.

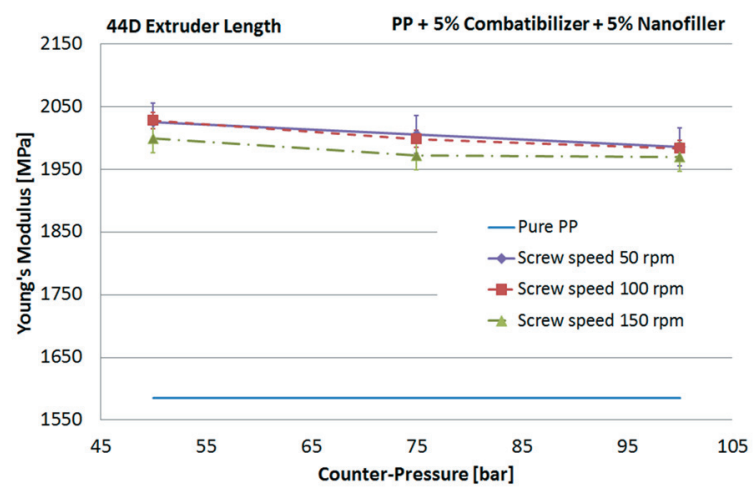

Fig. 2. Effects of screw speed and counter-pressure on Young's modulus using an extruder length of $44 D$

Fig. 3 shows the influence of screw speed and counter-pressure on Young's modulus at an extruder length of 52D. To explore only the effects of longer residence times, the additional $8 \mathrm{D}$ were only an 
elongation of the metering zone with conveying elements. In contrast to the results at the 44D extruder length, the processing conditions with a screw speed of $100 \mathrm{rpm}$ delivered the best Young's modulus values. Furthermore, there is no clear trend visible on the quality related to the counter-pressure. The residence time of the polymer melt varies between $260 \mathrm{~s}$ (100 bar), $245 \mathrm{~s}$ ( 75 bar) and $230 \mathrm{~s}$ (50 bar). It seems that a counter-pressure of 75 bar leads to the best results.

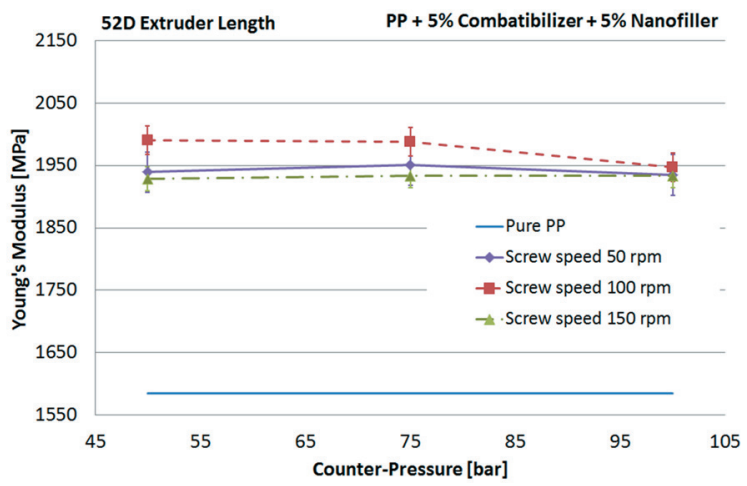

Fig. 3. Effects of screw speed and counter-pressure on Young's modulus using an extruder length of 52D

Clearly, the improvement in Young's modulus compared to virgin polypropylene was approximately $30 \%$ for the PNCs investigated, while the influence of processing conditions was low. A consolidated view indicates that longer residence time, which is certainly necessary for the diffusion process of intercalation and subsequent delamination of silicate platelets in the polymer matrix, seems to have a negative effect on the Young's modulus. The measurements with 52D extruder length led to worse results than the experiments with 44D. The influence of the screw speed controlled shear energy on the improvement of Young's modulus is not clear; further research is thus necessary.

\subsection{Effects of the Production with the Injection Moulding Compounder (IMC) on Young's Modulus}

Fig. 4 shows the influence of processing in a single heat. It can be clearly seen that the two-step process generates higher Young's modulus values than the processing in a single heat. This can have various reasons. The shear energy in the two-step process is higher than the introduced energy in the one-step process, because the pelletized compound has to be molten again in the injection moulding machine and so the injection screw introduces shear energy again. Another reason is that the residence time of the single heat process currently is 2.5 times higher than in the two-step process due to the PNC-IMC layout with its long-melt conveying system. Residence time in IMCs depends on the cycle time of the injection moulding machine and, therefore, it is variable to a certain extent. The two interacting mechanisms of exfoliation and degradation are difficult to separate; therefore, much further work is necessary to increase the degree of exfoliation without simultaneous material degradation.

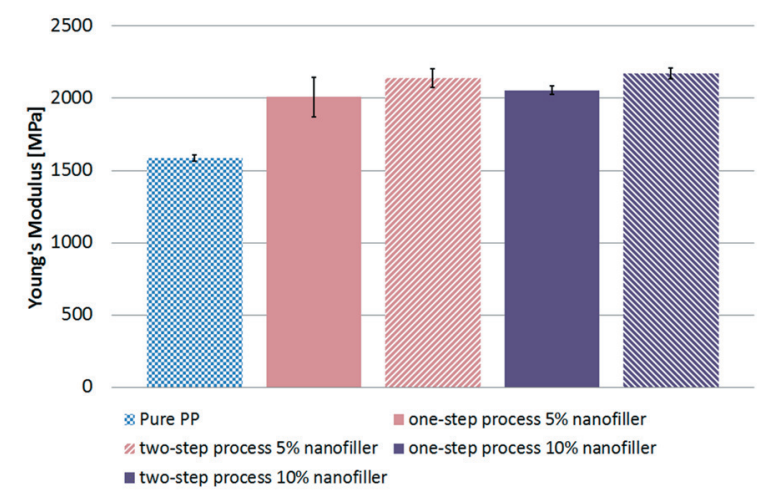

Fig. 4. Effects of one-step and two-step process on Young's modulus using an extruder length of $52 D$

Another important result of these measurements is that the amount of compatibilizer and nanofiller has remarkably little effect on Young's modulus. The increase up to $10 \mathrm{wt} \%$ improves the compounds stiffness less than $5 \%$.

\subsection{Effects of Additional Masterbatch Process on Young's Modulus}

The effect of an additional masterbatch process for the preparation of polypropylene nanocomposites is plotted in Fig. 5. Here, 3inl is the abbreviation for mixing PP, nanofiller and compatibilizer in one compounding process. This compounding procedure is compared with the masterbatch pre-stage process in which a masterbatch of $50 \mathrm{wt} \%$ organoclay and $50 \mathrm{wt} \%$ compatibilizer was produced in the twinscrew extruder and then pelletized. The masterbatch pellets were fed into the twin-screw extruder again and then diluted with PP to obtain a compound of 5 wt $\%$ nanofiller and $5 \mathrm{wt} \%$ compatibilizer. This new compound was either transported directly into the injection moulding machine (one-step process) or pelletized again and then injection-moulded (two-step process).

It is clear that the two-step process generates better Young's modulus values than processing in a single 
heat and that the masterbatch process shows immense potential for the production of nanocomposites. The increase in Young's modulus compared to virgin polypropylene is approximately $30 \%$ for $3 \mathrm{in} 1$, twostep process and one-step masterbatch process, whereas it is $40 \%$ for the masterbatch two-step process.

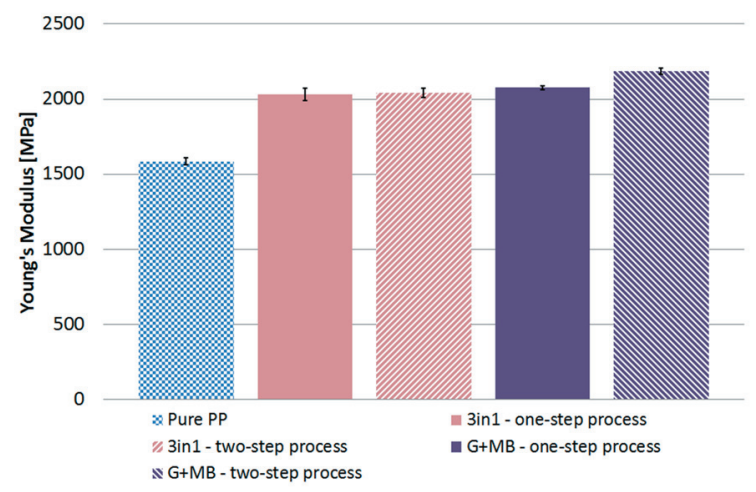

Fig. 5. Effects of additional masterbatch process in one-step and two-step processing on Young's modulus using an extruder length of $44 D$

In conclusion, all investigations of Young's modulus have shown that the conventional compounding with the pre-stage masterbatch process and the two-step process of the nanocomposite leads to the best values obtained thus far. Nevertheless, one should bear in mind that the 3in1 one-step process generates only $10 \%$ lower Young's modulus values than the conventional process but with savings in energy (up to $40 \%$ ) and time.

These results have also an essential aspect for the industry, because one of the biggest challenges remains the intercalation and subsequent delamination of silicate platelets in the polymer matrix. With the upstream masterbatch process stage, exploiting the full potential of nanofillers should be more easily achieved. Furthermore, a masterbatch is much easier to handle than a powder with a bulk weight of $210 \mathrm{~g} / 1$.

\subsection{Effects of Nanofillers at Various Pressures on Thermal Conductivity}

The increase in thermal conductivity and the influence of pressure on the thermal conductivity are shown in Fig. 6. To maintain an overview, only three different materials with 0,5 and $10 \mathrm{wt} \%$ at 0 and 750 bar were plotted. It can be clearly seen that the thermal conductivity strongly depends on pressure. The increase from 0 to 750 bar is about $10 \%$ in the molten state and about $25 \%$ in the solid state.
The increase of thermal conductivity through the use of nanofillers in the molten stage amounts about $7.5 \%$ with $10 \mathrm{wt} \%$ nanofiller and in the molten state about $10 \%$ related to the virgin PP. It is striking to see that the effect of $5 \%$ nanofiller in the solid state at 0 bar is much higher than at the 750 bar level.

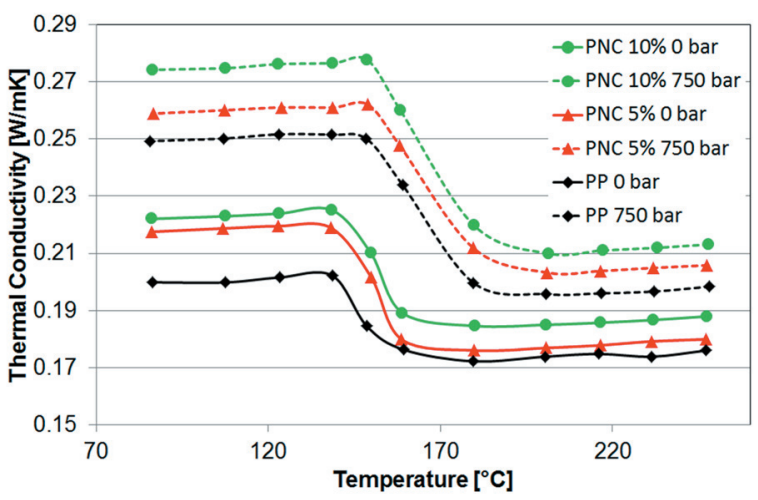

Fig. 6. Effects of nanofillers and pressure on thermal conductivity using an extruder length of $52 \mathrm{D}$

This is not actually a new phenomenon, but should be considered in the production of injection moulded parts regarding cycle time reduction and cost savings. As can be seen in the cooling time in Eq. (2), the thermal conductivity is linked via the effective thermal diffusivity in inverse proportion to the cooling time; therefore, an increase in thermal conductivity by $10 \%$ leads to a reduction of the cooling period by $10 \%$.

$$
t_{k}=\frac{s^{2}}{a_{e f f\left(T_{w}\right)} \cdot \pi^{2}} \cdot \ln \left[\left(\frac{4}{\pi}\right) \cdot\left(\frac{T_{M}-T_{W}}{T_{E}-T_{W}}\right)\right],
$$

where $t_{k}$ is cooling time, $s$ wall thickness, $a_{\text {eff(Tw) }}$ effective thermal diffusivity, $T_{M}$ melt temperature, $T_{W}$ mean cavity wall temperature and $T_{E}$ demoulding temperature. The increase of thermal conductivity by $10 \%$ still has potential to be improved towards $30 \%$. Therefore, focused research in this area towards better intercalation and subsequent delamination of silicate platelets in the polymer matrix will be performed in the near future.

\section{CONCLUSIONS}

The preparation of PP polymer nanocomposites has been carried out with an intermeshing, co-rotating twin-screw extruder of the PNC-IMC in order to improve Young's modulus and thermal conductivity; 11 or 13 barrel segments were used for the compounding process, and the PNC-IMC was used 
for processing of injected parts in a single heat, single plasticizing process.

As shown in this paper, there are many variables influencing Young's modulus and the thermal conductivity of polymer nanocomposites. Although it is possible to apply high shear forces and long residence time simultaneously, the results show that an extraordinarily long extruder (52D) and the additional use of a melt pump to generate counterpressure seems to have a negative effect on the improvement of Young's modulus. Additionally, high screw speed is not essential for the preparation of polymer nanocomposites. More effective regarding to the improvement of Young's modulus is the use of the pre-stage masterbatch process, where a masterbatch of $50 \mathrm{wt} \%$ nanofiller and $50 \mathrm{wt} \%$ compatibilizer is produced and further diluted with PP.

In conclusion, it is crucial to find a compromise between shear energy input and the residence time. Shear energy is necessary for intercalation and subsequent delamination of silicate platelets in the polymer matrix, while residence time is the most crucial parameter for the diffusion process; however, an overly high residence time may be critical for material degradation.

Currently, the chosen setup and process parameter set of the PNC-IMC production line enabled reaching the material property improvements of the two-step masterbatch process. To bring the one-step process beyond the two-step process, further research will be done on:

- modification of the screw geometry,

- perfect adaptation of the mixing ratio between nanofiller and compatibilizer,

- use of compatibilizers based on highly viscous PP-types, and

- special treatment of the PNC melt between extruder and melt accumulator (flow induced improvement of intercalation and exfoliation).

The results concerning the increase of thermal conductivity are encouraging and support further intensive investigations based on industrial interest.

\section{ACKNOWLEDGEMENTS}

We would like to thank Borealis, Linz for the provided PP, Dr. G. Pinter for the provided testing equipment and Dr. G.R. Berger for his valuable comments on the manuscript.

\section{REFERENCES}

[1] Jensen, R. (2001). Synergien intelligent genutzt. IMC-Spritzgießcompounder erhöht Wertschöpfung. Kunststoffe, vol. 91, p. 40-45.

[2] Sieverding, M., Bürkle, E., Zimmet, R. (2005). IMC Technology opens up new fields of application. Kunststoffe Plast Europe, vol. 8, p. 34-37.

[3] Bürkle, E., Scheel, G., Damedde, L. (2009). Energyefficient processing of natural fibre-reinforced plastics. Kunststoffe Plast Europe, vol. 2, p. 39-44.

[4] Wobbe, H. (2003). Trends und Visionen der Spritzgießtechnik. Kunststoffe, vol. 10, p. 60-65.

[5] Vaia, R.A., Jandt, K.D., Kramer, E.J., Giannelis, E.P. (1996). Microstructural evolution of melt intercalated polymer-organically modified layered silicates nanocomposites. Chemistry of Materials, vol. 8, no. 11, p. 2628-2635, DOI: $10.1021 / \mathrm{cm} 960102 \mathrm{~h}$.

[6] Mignoni, M.L., Silva, J.V.M., De Souza, M.O., Mauler, R.d.S., De Souza, R.F., Bernando-Gusmao, K. (2011). Polyethylene-montmorillonite nanocomposites obtained by in situ polymerization of ethylene with nickel-diimine catalysts. Journal of Applied Polymer Science, vol. 122, no.3, p. 2159-2165, DOI:10.1002/ app.34358.

[7] Kracalik, M., Laske, S., Gschweitl, M., Friesenbichler, W., Langecker, G.R. (2009). Advanced compounding: extrusion of polypropylene nanocomposites using the melt pump. Journal of Applied Polymer Science, vol. 113, no. 3, p. 1422-1428, DOI:10.1002/app.29888.

[8] Battisti, M.G., Friesenbichler, W. (2012). Injection molding compounding of PP polymer nanocomposites. Proceedings of International Conference PMI 2012, Ghent, p. 134-140.

[9] Sinha Ray, A., Okamoto, M. (2003). Polymer/ layered silicate nanocomposites: a review from preparation to processing. Progress in Polymer Science, vol. 28, no. 11, p. 1539-1641, DOI:10.1016/j. progpolymsci.2003.08.002.

[10] Kiliaris, P., Papaspyrides, C.D. (2010). Polymer/layered silicate (clay) nanocomposites: An overview of flame retardancy. Progress in Polymer Science, vol. 35, no. 7, p. 902-958, DOI:10.1016/j.progpolymsci.2010.03.001.

[11] Oswald, T.A., G. Menges (1996). Material Science of Polymers for Engineers. Carl Hanser Verlag Munich, Vienna, New York.

[12] Laske, S., Duretek, I., Witschnigg, A., Mattausch, H., Tscharnuter, D., Holzer, C. (2012). Influence of the degree of exfoliation on the thermal conductivity of polypropylene nanocomposites. Polymer Engineering \& Science, vol. 52, no.8, p. 1749-1753, DOI:10.1002/ pen.23121.

[13] Sibalija, T., Majstorovic, V., Sokovic, M. (2011). Taguchi-Based and Intelligent Optimisation of a MultiResponse Process Using Historical Data. Strojniški vestnik - Journal of Mechanical Engineering, vol. 57, no. 4, p.357-365, DOI:10.5545/sv-jme.2010.061. 